\title{
Pooled Human Immunoglobulin Preparations as Immunomodulating Drugs
}

\author{
T. L. Vassilev ${ }^{a, *}$ and O. V. Starkina ${ }^{a}$ \\ ${ }^{a}$ Institute of Biology and Biomedicine, Lobachevsky State University of Nizhny Novgorod, Nizhny Novgorod, 603950 Russia \\ *e-mail: tchavdarv@gmail.com
}

Received April 11, 2019; revised May 13, 2019; accepted May 15, 2019

\begin{abstract}
It is time to celebrate the 125th anniversary of the first successful attempt to develop and use a specific high-titer antitoxic serum for treating diphtheria, a deadly infectious disease. This was followed by major advances in passive immunotherapy 75 years ago (production of pooled human IgG for subcutaneous injection) and 50 years ago (widespread technology for producing immunoglobulin preparations for intravenous administration). More than 200 tons of pooled human IgG are produced per year worldwide. The preparation is used primarily for IgG substitution in patients with primary and secondary immunodeficiencies, as well as for an immunomodulating treatment of a growing number of autoimmune and inflammatory diseases. These preparations contain the pooled IgG antibody repertoire of a large population of healthy plasma donors. This repertoire includes antibodies that neutralize pathogens and their factors of virulence, anti-idiotypic antibodies, and antibodies to other foreign and own proteins, as well as to carbohydrate antigens. Naturally polyspecific antibodies that are present in all healthy individuals play an important role as a first-line defense against bacteria and viruses. After exposure to protein-modifying agents, some IgG molecules can acquire the ability to bind novel structurally unrelated antigens. This phenomenon is referred to as induced polyspecificity. The list of these protein-modifying molecules was shown to include low-pH buffers, free heme, pro-oxidative ferrous ions, reactive oxygen species, etc. Such modified antibody preparations may have a therapeutic potential, since their administration to animals with experimental sepsis or aseptic systemic response syndromes significantly improved survival rates, while the same dose of the native preparation had no effect. We also hypothesize that the aggressive protein-modifying molecules released in sites of inflammation and tissue damage could also modify the antigen-binding behavior of surface immunoglobulin B cell receptors and the structurally related T cell receptors. This "specificity editing" of both types of receptors may play a major role in the body's defense mechanisms.
\end{abstract}

Keywords: IgG, polyspecific antibodies, passive immunotherapy, sepsis, systemic inflammatory response syndrome

DOI: $10.1134 / \mathrm{S} 0026893319050145$

\section{YEARS OF PASSIVE IMMUNIZATION}

The first microorganisms causing infectious diseases were described at the end of the 19th century, in the same period that the first attempts to develop specific methods of treatment were undertaken. By administering horses, goats, and guinea pigs increasing doses of diphtheria and tetanus toxins, Emil von Behring, Paul Ehrlich, and their colleagues found that, after a certain period of time, the serum of the experimental animals acquired the ability to prevent and cure the same diseases when injected to other non-immune animals. The first article describing the effects of diphtheria and tetanus antitoxins dates back to 1890 . The initial attempts to treat human patients

Abbreviations: CCHF, Crimean-Congo hemorrhagic fever; IVIg, intravenous immunoglobulin G; LPS, lipopolysaccharide; SIRS, systemic inflammation response syndrome; ROS, reactive oxygen species. using the same animal sera were unsuccessful. However, the issues related to antitoxin standardization and production were rapidly resolved, and after 1894, animal anti-diphtheria serum was successfully applied worldwide in the treatment of children with this deadly infectious disease. In the first decades of the 20th century, specific high-titer animal immunoglobulins were developed to treat patients with many other infectious diseases. This treatment had obvious and inevitable side effects: all patients became sensitized to animal proteins, and repeated administration of the same preparation was dangerous.

It may seem surprising that high-titer specific animal immunoglobulins are still produced and administered in the 21th century. In fact, the reason is simple: very few patients currently require specific antibody preparations for passive immunotherapy of diphtheria, tetanus, gas gangrene, snake venom poisoning, or botulinum intoxication. Biotechnology companies are 
not interested in producing specific human immunoglobulins or humanized monoclonal antibodies with the same specificity because of the very low demand, which is why preparations of the plasma of immunized animals are still in use.

The next important development in the history of passive immunotherapy of infectious diseases occurred 80 years ago. Technologies for the large scale fractionation of human plasma proteins were developed by Edwin Cohn et al. [1] in the United States during World War II to enable the industrial production of human serum albumin for treating wounded soldiers. It was shown that the plasma component known as fraction II contained fairly pure human IgG; since then, this fraction has been used to obtain IgG for subcutaneous administration for the purposes of passive immunoprophylaxis and immunotherapy of several infectious diseases.

The first attempts of administering human serum albumin or human IgG intravenously had unexpected catastrophic consequences. All patients who were administered human serum albumin developed "jaundice", later described as viral hepatitis. This problem was solved quite rapidly by using albumin preparations only after a 10 -h-long incubation at $60^{\circ} \mathrm{C}$. Intravenous infusions of the total IgG from Cohn's fraction II caused a severe drop of blood pressure, chills, and hyperpyrexia in all patients. Another 20 years were required to determine the mechanisms of these unexpected side effects and find ways to avoid them. It turned out that their principal cause was the presence of the aggregated $\mathrm{IgG}$ molecules in the preparations for intravenous administration. These aggregates lead to complement activation, and anaphylatoxins $\mathrm{C} 3 \mathrm{a}$ and C5a produced as a result caused a rapid drop of blood pressure. In the 1960s, several techniques were developed to reduce the presence of $\mathrm{IgG}$ aggregates and suppress their formation during the shelf-life period.

Each batch of therapeutic intravenous immunoglobulin (IVIg) must meet the requirements comprehensively described in the current National or European Pharmacopoeia. These requirements can be summarized as follows:

- only the plasma of healthy donors can be used for fractionation;

- the preparation should contain at least $90 \%$ of $\mathrm{IgG}$ monomers and dimers and only a minor fraction of aggregated IgG molecules; moreover, IgG molecules must not be modified: they must be in their native state and possess normal $\mathrm{F}(\mathrm{ab})_{2}{ }^{-}$and $\mathrm{Fc}$-fragment-dependent biological functions;

- the distribution of $\operatorname{IgG}$ subclasses should be the same as in the plasma of healthy adults, and the IgA content should be low;

- the activity of prekallicrein activator (PKA), the ability to activate the complement system, and the concentrations of plasmin, isohemagglutinin, and autoanitbodies (anti-D and other) must be below the predefined threshold levels;

- the presence of preservatives (such as merthiolate) is not allowed;

- the titers of certain specific (antiviral and antibacterial) antibodies should be above the specific guaranteed levels.

\section{ANTIBODY REPERTOIRES OF POOLED THERAPEUTIC IgG}

Each production batch of human immunoglobulins is fractionated from the pooled plasma of more than 1000 healthy donors (unlike the term pooled $\operatorname{Ig} G$ used worldwide, the Russian term normal human immunoglobulin for intravenous administration does not reflect combined plasma of numerous donors as the origin of the preparation). The plasma is obtained from donor blood after removing the erythrocytes and other cells. An alternative technique is plasmapheresis, a procedure in which the plasma is collected and utilized, while the blood cells are returned into the donors' bloodstream. Thus, a preparation obtained from normal plasma contains a repertoire of antibodies of a large disease-free human population. New techniques, such as phage libraries or microarrays, make it possible to analyze in detail the repertoire of antiprotein and frequently ignored antiglycan antibodies.

While all $\operatorname{IgM}$ antibodies are polyreactive, only some $\operatorname{IgG}, \operatorname{IgA}$, and IgE molecules can bind several structurally different antigens. The formal proof that such antibodies do exist came from the early efforts to produce monoclonal antibodies. To the disappointment of researchers who expected the constructed hybridoma to produce only antibodies highly specific to the antigen they injected in mice, the hybridomas frequently secreted polyspecific antibodies. An antibody is classified as polyspecific if it has been demonstrated to bind at least two structurally different foreign antigens or autoantigens. Nevertheless, it cannot be ruled out that any antibody could be found to interact with two or more antigens if sufficiently large antigen panels were tested [2].

Polyspecific antibodies are often neglected as "background," "silent" or "sticky." Few immunologists are interested in their biological role. However, there are convincing data that suggest their importance as the first line of defense against an invasion of pathogenic microorganisms [3]. This type of polyspecificity is termed natural or innate [4]. Antibodies can also acquire antigen-binding polyspecificity after contact with protein-modifying molecules in vitro or in vivo in the sites of inflammation, as discussed below.

Idiotypic interactions of individual antibody molecules with anti-idiotypes are responsible for the formation of IgG dimers in IVIg preparations. Since the 
affinity of these interactions is low, dimers are formed only after several months of storage at $4^{\circ} \mathrm{C}$. According to the "network" hypothesis proposed by N.K. Jerne, idiotype-anti-idiotype interactions play a central role in the self-regulation of the immune system [5]. Subsequently, it was shown that the control mechanisms do not depend strictly on the idiotype, and the network concept gradually lost its appeal, although this possibility should not be forgotten. For instance, a single IVIg administration to patients with autoimmune hemophilia resulted in a rapid disappearance of $95 \%$ of the disease-associated $\mathrm{IgG}$ antibodies against human factor VIII. It was shown that this effect was due to the anti-idiotypic antibodies of the IgG preparation binding with idiotypes of the patient's pathological IgG antibodies against factor VIII [6].

The repertoire of IVIg specificities was found to include numerous autospecificities. Their presence has nothing to do with pathological autoimmunity. Detection techniques such as ELISA, immunoblotting, or microarrays employ immobilized self- proteins and allow binding of low-affinity autoantibodies that have no pathological relevance. In some rare cases, the presence of plasma antibodies that are not organ-specific could be a predictor marker for a future autoimmune disease. For example, the presence of IgG antibodies to double-stranded DNA in a healthy individual is a sign to expect a full-blown rheumatic disease [7].

\section{POOLED HUMAN IgG PREPARATIONS FOR INTRAVENOUS ADMINISTRATION FOR ANTIBODY SUBSTITUTION AND FOR TREATMENT OF AUTOIMMUNE DISEASES}

The worldwide production of therapeutic human IVIgG increases each year. Most of it is used to treat three groups of patients: those with primary immune deficiency, secondary immune deficiencies, and autoimmune and inflammatory diseases.

Regular subcutaneous or intravenous administration of a pooled immunoglobulin preparation is the main and often the only life-saving treatment of most primary immunodeficiencies. More than 300 of them are currently known, and this number is growing. The first one to be described is Bruton agammaglobulinemia. It is an X-linked recessive disease characterized by low plasma immunoglobulin levels and very low B cell numbers. It develops as a result of mutations in Bruton's tyrosine kinase. With regular IgG-replacement therapy, the patient's life expectancy and quality of life can be close to normal.

The most widespread type of primary immune deficiency is common variable immune deficiency (CVID). Approximately 10-20\% of patients have a monogenic disorder, which may exhibit autosomal recessive or autosomal dominant inheritance. Along with frequent infections, clinical manifestations of
CVID include chronic obstructive pulmonary disease, bronchiectasis, diarrhea, and a high frequency of autoimmune diseases. In addition to IgG-replacement therapy, the treatment may include antibiotics, parenteral nutrition, stem cell transplantation, etc.

Patients with primary immune deficiency are significantly less numerous than those with secondary immune deficiency. The causes of the latter include (but are not limited to) the following pathological conditions: multiple myeloma, chronic lymphoblastic leukemia, other hematologic malignancies, solid malignant tumors, low B-cell levels after rituximab treatment, lack of response to antibiotic therapy, premature birth, geriatric immune deficiency, etc.

Many countries currently face a deficiency of IVIg preparations, mainly because the numbers of patients with autoimmune diseases treated with high IVIg doses are rapidly growing. This problem is comprehensively discussed in one of the recent reviews [8].

\section{SPECIFIC POLYCLONAL IMMUNOGLOBULINS FOR INTRAVENOUS ADMINISTRATION}

Some companies that perform fractionation of human plasma produce small batches of high-titer specific immunoglobulins for passive immunization or treatment of certain infectious diseases, in particular, rabies, tetanus, hepatitis B, varicella, cytomegalovirus, and tick-borne encephalitis.

Thirty years ago, the National Center of Infectious and Parasitic Diseases (Bulgaria) developed and clinically tested an intravenous immunoglobulin preparation for treating Crimean-Congo hemorrhagic fever (CCHF-Venin). The immunoglobulin was obtained from the plasma of volunteers immunized with the $\mathrm{CCHF}$ vaccine. Previously, the same hyperimmune plasma had been used to produce high-titer specific anti-CCHF immunoglobulin for subcutaneous passive immunization. However, its administration to patients with a severe disease was found to cause strong life-threatening hemorrhage at the site of injection. An urgent need for a preparation with the same specificity that could be administered intravenously was obvious. In the summer of 1989, seven patients with severe $\mathrm{CCHF}$ were administered a single intravenous injection of CCHF-Venin. This study did not contain any control group, because no other drug for CCHF treatment was available at the time. In all subjects, the prognosis was poor, but after the treatment they rapidly recovered and were dismissed from the hospital [9]. Unfortunately, the company that manufactured the first batch of CCHT-Venin was not interested in producing it on a regular basis because of the lack of financial incentive. As a result, CCHT-Venin became part of the sad list of life-saving "orphan" drugs that no pharmaceutical or biotech company wishes to produce. 


\section{EXPERIMENTAL POLYCLONAL IgM AND IgA IMMUNOGLOBULIN PREPARATIONS}

It is known that IgM antibodies are naturally polyspecific. The potent immunomodulating activity of total human IgM is well described and has an obvious therapeutic potential $[10,11]$. However, none of the companies that perform plasma fractionation have succeeded in producing an intravenous immunoglobulin preparation with a high IgM content. For instance, Biotest (Germany) manufactures BT086 product that is enriched in IgM/IgA. It contains $23 \%$ IgM while the remaining immunoglobulins are $\mathrm{IgG}$ and IgA. The first clinical trials of this drug marketed under the name of Trimodulin were conducted in patients with severe community-acquired pneumonia who were in need for the invasive procedure of artificial pulmonary ventilation [12]. In these trials Trimodulin did not have a therapeutic effect, probably because of the low IgM content.

There have also been attempts to utilize human serum IgA. Some of these immunoglobulins exist as dimers, and secretory antibodies similar to natural ones can be obtained by adding a secretory component $[13,14]$. We could not find evidence that these antibodies have been already tested in clinical trials.

It can be expected that techniques of dimerization of monomeric human serum $\operatorname{IgA}$ will be developed in the nearest future. Addition of secretory component to these molecules will make them identical to natural secretory IgA. There are at least two important reasons to pursue this research direction. Firstly, too many newborn children are bottle-fed now with "humanized" (actually, cow) milk. They would strongly benefit if their digestive system is protected by the addition of human secretory IgA to the non-human milk. The second beneficiaries will be human plasma fractionation companies. Human plasma is a very precious raw material and the ability to use a fraction that had been thrown away before will be a very positive development.

\section{INDUCED ANTIBODY SPECIFICITY: EXPOSURE TO AN ACIDIC BUFFER MODIFIES THE ANTIGEN-BINDING AND BIOLOGICAL PROPERTIES OF IgG ANTIBODIES}

It has been two decades since the phenomenon of induced antibody specificity was described [3, 4]. Some monoclonal $\mathrm{IgG}$ and $\mathrm{IgE}$ antibodies, as well as all preparations of the total (polyclonal) antibodies analyzed, acquired an ability to bind to broader antigen panels after treatment with different protein-modifying agents. The range of such protein-modifying treatments includes exposure to acidic $\mathrm{pH}$ and free heme, as well as $\mathrm{Fe}(\mathrm{II})$ compounds or reactive oxygen species (ROS) bacterial.
The study by Bouvet et al. [15] was the first one to report the effect of acidic buffers on immunoglobulin preparations. This phenomenon cannot be related directly to events occurring in vivo. However, the production of some licensed therapeutic IVIg preparations involves a stage of protein fractionation at low $\mathrm{pH}$, which enhances their antigen-binding polyspecificity and alters their biological properties. A comparative study of the effects of different IVIg preparations used for passive immunotherapy of sepsis in a mouse model showed that administration of native IVIg (i.e., without treatment with an acidic buffer) did not affect the survival rate, whereas a single dose of the same preparation treated with a buffer with $\mathrm{pH} 4.0$ significantly decreased the mortality rate due to the septic shock induced by lipopolysaccharides (LPS). This important observation makes it clear that different commercially available immunoglobulin preparations may possess different antigen-binding and therapeutic characteristics. There is every reason to expect that preparations with enhanced polyspecificity will have a therapeutic potential in patients with different variants of the systemic inflammation response syndrome (SIRS).

The molecular modifications of immunoglobulins exposed to $\mathrm{pH} 4.0$ have been partially elucidated [16, 17]. The one-step method of choice for the rapid purification of $\mathrm{IgG}$ of human and animal origin is based on the specific binding of molecules of this immunoglobulin isotype to immobilized Protein A or Protein $\mathrm{G}$. The elution is performed by washing of the respective immunoaffinity columns with a $\mathrm{pH} 2.8$ buffer. Our studies have shown that this treatment dramatically changes the structures of the $\mathrm{IgG}$ molecules and causes a strong enhancement of IgG antigen-binding polyspecificity that comes close to unspecific stickiness. This IgG purification method has to be avoided and replaced by more mild techniques (e.g. with the Gentle $\mathrm{Ag} / \mathrm{Ab}$ Elution Buffer from ThermoFischer Scientific-see https://www.thermofisher.com/order/ catalog/product/21004).

\section{HEME-INDUCED ANTIBODY POLYSPECIFICITY}

Heme is a macrocyclic iron-containing compound that serves as a prosthetic group of numerous proteins involved in gas transport, oxidative metabolism, and cellular signaling. It is also known that heme participates in different cellular processes, including transcription, translation, and cell differentiation. At the same time, the strong redox activity characteristic for heme might be a source of danger, especially if heme is released from the protein-bound state. Under various pathological conditions, such as hemolysis, ischemia-reperfusion injury, hemorrhage, or rhabdomyolysis, large amounts of heme-containing proteins can be released into the circulation. These proteins easily lose their prosthetic group extracellularly. In plasma 
there are several heme-sequestering proteins such as hemopexin and albumin that are involved in its binding and in preventing the free heme toxicity. However, in conditions of excessive release of hemoproteins, heme-scavenging proteins can be saturated and the plasma concentration of free heme may reach more than $20 \mathrm{mM}$. As immunoglobulins are one of the most abundant plasma proteins they may well encounter free heme in the bloodstream. McIntyre et al. [18] showed that exposure of healthy donor immunoglobulins to heme induced new activities against phospholipids and many other autoantigens. The authors observed that heme-exposed antibodies of healthy donors bound phospholipids in the same manner as autoantibodies obtained from patients with the antiphospholipid syndrome. Along with anti-autoantigen activity, heme-exposed immunoglobulins exhibited antibacterial activity against individual bacterial antigens and against intact bacteria [19]. The fact that heme-exposed IgG effectively binds bacterial antigens explains its ability to initiate complement-mediated disruption of bacteria. Pathogen opsonization by antibodies with induced polyspecific reactivity can trigger the precipitation of complement fragments and enhance their immunogenicity, as previously assumed for polyreactive antibodies. It should be noted that some pathogenic bacteria induce hemolysis to obtain heme as a vital iron-containing growth factor. The development of novel antibacterial specificity in the heme-sensitive fraction of antibodies represent a mechanism for delaying the dissemination of hemolytic bacteria until the adaptive immune response takes over.

We speculate that in certain pathological conditions, heme-mediated generation of new antigenbinding specificities may have harmful effects. For instance, ischemia-reperfusion syndrome is a severe inflammatory reaction that leads to tissue damage and is mediated mainly by incorrect activation of the complement system. It was recently shown that native antibodies are responsible for the initial complement activation in ischemia-reperfusion. The initial hypoxia and the release of reactive oxygen species (ROS), of heme and/or of transition redox-active metals may result in cellular damage and in exposure of cryptic epitopes on the cell surface. These epitopes are then recognized by circulating natural antibodies. The clustering of antibodies to the cell surface results in complement activation and subsequent tissue damage. It cannot be ruled out that heme and ROS affect not only cell surface proteins but also the immunoglobulins present in the bloodstream. Thus, exposure to redox-active compounds released in ischemia may generate novel antigen-binding properties in a fraction of locally circulating antibodies. This would result in the binding of these antibodies to cell surface autoantigens, followed by an activation of the complement system and tissue damage.
AN URGENT NEED FOR NOVEL THERAPEUTIC APPROACHES IN SEPSIS AND THE THERAPEUTIC POTENTIAL OF IMMUNOGLOBULIN PREPARATIONS WITH ADDITIONALLY ENHANCED POLYSPECIFICITY

Sepsis is caused by a severe, generalized and uncontrolled systemic inflammation in response to invading pathogens resulting in a multiple organ dysfunction. It is a major killer worldwide. In the United States alone, more than 750000 cases of severe sepsis are recorded annually, and over 210000 of them have a lethal outcome. A less-known fact is that many of the surviving patients remain disabled because of immunological disorders, cognitive dysfunctions of varying severity, anemia, etc. More than a half of them die within the following five years [20-22].

It was recently shown that the mechanisms that underlie severe traumatic aseptic inflammation syndrome resemble those involved in sepsis. As a result of severe trauma, damage-associated molecular patterns (DAMPs), such as formyl peptides and mitochondrial DNA, are released into the bloodstream and activate the innate immune response. Both compounds activate polynuclear neutrophils via formylpeptide receptor 1 and Toll-like receptor 9 (TLR9) [23]. As a result of trauma, large amounts of free heme are frequently released into the bloodstream, where heme binds to TLR 4 cell receptors and causes a strong proinflammatory response similar to that induced by bacterial LPS [24].

Currently, there are no drugs for the specific therapy of patients with sepsis and aseptic systemic inflammatory response syndrome (SIRS). Even the administration of monoclonal antibodies agains individual proinflammatory molecules does not affect survival. A recent study in SIRS patients revealed a significant modification of the expression patterns of over $80 \%$ of their genes; the authors referred to this effect as a genomic storm [25]. The genomic storm phenomenon probably explains the failure of attempts to improve the outcome by neutralizing individual proinflammatory mediators. Obviously, when so many things go wrong simultaneously, the selective elimination of a single disease-associated inflammationrelated molecule does not make much difference. There is a need for a therapeutic agent with a broad neutralizing potential, and IVIg is a promising candidate. These are polyspecific therapeutic agents that are known to neutralize a broad spectrum of pathogens as well as their virulence factors. Moreover, IVIg preparations exhibit antiinflammatory and immunomodulating properties; this effect is well established, although insufficiently understood. However, in their present form, they have not been really successful in preventing sepsis-induced death [26].

The number of infectious diseases where a severe generalized inflammatory reaction plays a major role in elevated mortality is constantly growing. Influenza 
caused by the highly pathogenic strains of influenza virus $\mathrm{A}$ (H1N1 in 1918 and $\mathrm{H} 5 \mathrm{~N} 1$ in 2009) and some hemorrhagic fevers are among the recent additions to this list [27-30].

\section{THERAPEUTIC IMMUNOGLOBULINS MODIFIED BY EXPOSURE \\ TO PRO-OXIDATIVE MOLECULES BECOME PROTECTIVE IN EXPERIMENTAL SEPSIS}

It has been shown previously that the in-vitro exposure of some IgG antibodies to ferrous ions or to ROS enhanced their antigen-binding polyspecificity that included binding to at least one pro-inflammatory cytokine [31]. The in-vivo $\operatorname{IgG}$ recirculation through an inflamed tissue has the same effect [32]. After a preliminary prooxydant treatment in vitro, IVIg preparations were used for passive immunotherapy of experimental sepsis in mice. Sepsis was induced by injecting bacterial LPS, live Escherichia coli, or zymosan, or by puncture and ligature of the cecum. In contrast to commercially available native IVIg preparations, a single dose of modified Ig significantly increased the survival of animals in all experimental models of sepsis [33].

The mechanisms of the observed protective activity of the IVIg modified by ferrous ions exposure were studied in detail in LPS-induced systemic inflammation. Its therapeutic effect was not due to a more efficient neutralization of LPS and was still present when administered one hour after LPS. The serum levels of several mediators of inflammation were decreased, IL10 levels were enhanced, the complement component $\mathrm{C} 3$ exhaustion was diminished and the coagulation abnormality was overcome [33]. Treatment with $\mathrm{Fe}(\mathrm{II})$ ions induced structural modifications in the antigen-binding site of $\mathrm{IgG}$ molecules, as indicated by the data of fluorescence spectroscopy, as well as kinetic and thermodynamic analyses. Apparently, these changes did not involve denaturation, because the modified preparations still met the strict pharmacopeial requirement for IVIg (Djoumerska-Aleksieva et al., manuscript in preparation). We expect that this improved IVIg will be effective in treating sepsis and other SIRS variants (e.g., posttraumatic, caused by avian influenza, etc.).

So far, the exact mechanism of the protective effect of IVIg with additionally induced polyspecificity has been subject to different hypotheses. Since IVIg affects multiple systems and in different ways (for example, FcR binding, catabolism of endogenous IgG, idiotypic interactions both with serum immunoglobulins and with B cell receptors, binding numerous self-antigens with a special affinity to a number of cytokines and membrane receptors, etc.), it is difficult to pick a favorite speculation. Nevertheless, considering the entire body of known facts, we favor two hypotheses. One is as straightforward, mechanistic and reductionist as any contemporary frontend immunology while the other is with a touch of philosophy in the best traditions of the European school. It should be pointed out straight away that both hypotheses (as well as many others) are not mutually exclusive.

The first hypothesis is based on a recent discovery by Rauch et al. [34], who found that a small subpopulation of mouse B1 cells, referred to as B1 innate response activators (IRA B1), migrates to the spleen, secretes GM-CSF, and controls the cytokine storm in sepsis. B1 cells are not only natural poly/autoreactive B cells but also represent he main source of idiotypically connected antibodies. It is intriguing to speculate a link between the polyspecificity and idiotypic connectivity of IVIg, the role of immunoglobulins as the preferred self-antigens in serum for IVIg polyreactivity and the respective properties of $\mathrm{B} 1$ cells. The logical inference is that B1 cells would be a preferred cellular target when idiotypic interactions are considered. That is why this recent report by Rauch et al. points to a possible mechanism of sepsis control by IVIg preparations with induced polyspecificity. Indeed, our preliminary results show that IRA B1 cells, themselves good binders of IVIg, show by far the strongest increase in IVIg binding after induction of polyspecificity by ferrous ions. Thus, it is possible that Fe-exposed IVIg targets this population, including by $\mathrm{B}$ cell receptor idiotypic interactions and, in the context of the present endotoxin, activates and augments their function. It is possible that this is how IVIg controls the cytokine storm in sepsis. This hypothesis is currently being tested.

At the same time, from other experiments in our lab it became clear that, while levels of inflammatory cytokines are reduced by the Fe-exposed IVIg treatment as compared to treatment with native (unmodified) IVIg, the levels of IL-10 were transiently increased. Both these effects bear direct relevance to the course of sepsis. While the IL-10 increase may be directly related to the observed binding to B1 cells (not only IRA) as well as to marginal zone B cells in the spleen, the decrease of IL- 6 and TNF $\alpha$ may be due to a variety of mechanisms. We favor the one suggested by Avrameas and Coutinho 30 years ago [35]. They proposed a role for the polyspecific binding of cytokines in the immunoregulatory effects of natural antibodies. This brilliant, but also strikingly speculative hypothesis did not become particularly popular, but it explains well the effects observed by us. It was this hypothesis, with one important amendment, that became the foundation of our principal concept. If natural antibody reactivities are meant to limit cytokine activities, why doesn't this compromise their signaling function? Perhaps, unlike Fe(II)-exposed IVIg, the natural buffer of the cytokine storm is represented by IgM natural antibodies that circulate in the plasma compartment only and would limit the systemic increase of cytokine levels, but would not affect their local function in tissues. Natural antibodies are mainly of the IgM isotype. They are produced by B1 cells, and their repertoire is positively selected on self-antigens 
during the fetal period. IgG antibodies, on the other hand, are mostly derived from B2 cells and belong to a repertoire selected by a strong negative selection stage. It is possible that the buffering function is an evolutionary adaptation specific for the B1-derived IgM repertoire. It would serve to limit cytokine action only in the vascular bed, where the inflammatory cytokines are more trouble than help. Their uncontrolled increase in the serum actually represents to a great extent the said "cytokine storm." The artificially inducible polyreactivity in B2-derived $\mathrm{IgG}$ antibodies renders the same property onto the isotype that is meant to penetrate the tissues (Fig. 1). In physiological conditions that would be undesirable and evolution has avoided it. During sepsis, though, this seems very helpful.

\section{INDUCED ANTIBODY POLYSPECIFICITY, IMMUNE REGULATION, INNATE IMMUNITY AND AUTOIMMUNITY}

The biological role of polyreactive antibodies may not be limited to their contribution to the first-line defense against pathogens in the pre-immune host $[36,37]$. Evidence from both in vitro and in vivo experiments, that have been reviewed above, shows that inflammation can increase the polyspecificity of some circulating IgG antibodies. It is logical to go further and to suggest that the aggressive molecules released in sites of inflammation could also modify the structure and in result - the antigen-binding behavior of surface immunoglobulin BCRs. If this modification is not accompanied by impairing the viability and long-term survival chances of the B-lymphocytes themselves, the consequences could be very far-reaching. The appearance of novel, previously "masked" specificities on membrane $B$ cell receptors would result in activation of B lymphocytes that would otherwise remain unresponsive. The ensuing proliferation, clonal selection, somatic hypermutation and affinity maturation of the activated B cells would lead to a skewing of the repertoire of locally available B-cell receptors, as well as of circulating antibodies, that may be better adapted to control this particular infection. This putative mechanism, which we call specificity editing may lead to a rapid and dramatic expansion of the spectrum of antigens of invading pathogens recognized by the circulating antibodies and by the locally present immune cells. The specificity editing of some B-cell receptors in combination with the simultaneous editing of B-cell receptor genes will rapidly generate new antigen-biding specificities, which themselves will increase the efficiency of the defense against a microorganism previously unknown to the immune systems The specificity of the newly-acquired antigen-binding is unpredictable. It could be either anti-foreign- or anti-self-antigen directed. As we have hypothesized previously, such inflammation-induced "specificity-editing" should take place outside primary lymphoid organs,

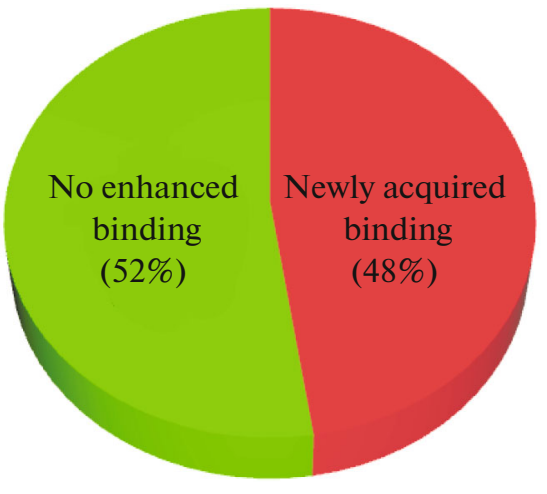

Fig. 1. Following exposure to Fe(II), IVIg becomes capable of binding with nearly a half of all randomly selected inflammation-related molecules (see [33]).

i.e. where the processes of negative selection of potentially harmful autoreactive B-cell clones are not operational. This may lay the foundation for the development of pathological autoimmune processes and thus contribute to explaining the link between infection and autoimmunity [31, 38].

Ferrous ions, ROS, heme, and probably other aggressive self-oxidation activators released at the sites of inflammation, traumatic lesions, and hemorrhages may well have an effect beyond the range of circulating immunoglobulins and antigen-binding cell receptors. These activators may also affect the molecular structure and the binding specificity of the complement components, of the coagulation and fibrinolysis cascades, as well as hormones, growth factors, and cytokines and their receptors, which would also change their biological properties. If this proves to be the case, inflammation could have important, previously unsuspected effects on maintaining antigenic homeostasis and on the body defense mechanisms.

\section{FUNDING}

This review did not require any special funding.

\section{COMPLIANCE WITH ETHICAL STANDARDS}

The authors declare that they have no conflict of interest. This article does not contain any studies involving humans or animals as experimental objects.

\section{REFERENCES}

1. Cohn E.J., Oncley J.L., Strong L.E., Hughes W.L., Armstrong S.H. 1944. Chemical, clinical and immunological studies on the products of human plasma fractionation: 1 . The characterization of the protein fractions of human plasma. J. Clin. Invest. 23, 417-432.

2. Van Regenmortel M.H.V. 2014. Specificity, polyspecificity, and heterospecificity of antibody-antigen recognition. J. Mol. Recognit. 27, 627-639. 
3. Dimitrov J. D., Planchais C., Roumenina L.T., Vassilev T.L., Kaveri S.V., Lacroix-Desmazes S. 2013. Antibody polyreactivity in health and disease: Statu variabilis. J. Immunol. 191, 993-999.

4. Kanyavuz A., Marey-Jarossay A., Lacroix-Desmazes S., Dimitrov J.D. 2019. Breaking the law: Unconventional strategies for antibody diversification. Nat. Rev. Immunol. http://doi.org/10.1038/s41577-019-0126-7

5. Jerne N.K. 1974. Clonal selection in a lymphocyte network. Soc. Gen. Physiol. Ser. 29, 39-48.

6. Rossi F., Dietrich G., Kazatchkine M.D. 1989. Antiidiotypic suppression of autoantibodies with normal polyspecific immunoglobulins. Res. Immunol. 140, 19-31.

7. Elnady B.M., Kamal N.M., Shaker R.H., Soliman A.F., Hasan W.A., Alghamdi H.A., Algethami M.M., Jajah M.B. 2016. Prevalence and clinical significance of nonorgan specific antibodies in patients with autoimmune thyroiditis as predictor markers for rheumatic diseases. Medicine (Baltimore). 95, e4336.

8. Perez E.E. Orange J.S., Bonilla F., Chinen J., Chinn I.K., Dorsey M., El-Gamal Y., Harville T.O., Hossny E., Mazer B., Nelson R., Secord E., Jordan S.C., Stiehm E.R., Vo A.A., Ballow M. 2017. Update on the use of immunoglobulin in human disease: A review of evidence. J. Allergy Clin. Immunol. 139, S1-S46.

9. Vassilenko S.M., Vassilev T.L., Bozadjiev L.G., Bineva I.L., Kazarov G.Z. 1990. Specific intravenous immunoglobulin for Crimean-Congo haemorrhagic fever. Lancet. 335, 791-792.

10. Hurez V., Kazatchkine M.D., Vassilev T., Ramanathan S., Pashov A., Basuyaux B., de Kozak Y., Bellon B., Kaveri S.V. 1997. Pooled normal human polyspecific IgM contains neutralizing anti-idiotypes to IgG autoantibodies of autoimmune patients and protects from experimental autoimmune disease. Blood. 90, 40044013.

11. Vassilev T., Mihaylova N., Voynova E., Nikolova M., Kazatchkine M., Kaveri S. 2006. IgM-enriched human intravenous immunoglobulin suppresses $\mathrm{T}$ lymphocyte functions in vitro and delays the activation of T lymphocytes in hu-SCID mice. Clin. Exp. Immunol. 145, 108-115.

12. Welte T., Dellinger R.P., Ebelt H., et al. 2018. Efficacy and safety of trimodulin, a novel polyclonal antibody preparation, in patients with severe community-acquired pneumonia: A randomized, placebo-controlled, double-blind, multicenter, phase II trial (CIGMA study). Intensive Care Med. 44, 438-448.

13. Longet S., Miled S., Lötscher M., Miescher S.M., Zuercher A.W., Corthésy B. 2013. Human plasma-derived polymeric IgA and IgM antibodies associate with secretory component to yield biologically active secretorylike antibodies. J. Biol. Chem. 288, 4085-4094.

14. Longet S., Vonarburg C., Lötscher M., Miescher S., Zuercher A., Corthésy B. 2014. Reconstituted human polyclonal plasma-derived secretory-like $\operatorname{IgM}$ and $\operatorname{IgA}$ maintain the barrier function of epithelial cells infected with an enteropathogen. J. Biol. Chem. 289, 2161721626.

15. Bouvet J.P., Stahl D., Rose S., Quan C.P., Kazatchkine M.D., Kaveri S.V. 2001. Induction of natural autoantibody activity following treatment of human im- munoglobulin with dissociating agents. J. Autoimmun. 16, 163-172.

16. Djoumerska-Alexieva I.K., Dimitrov J.D., Voynova E.N., Lacroix-Desmazes S., Kaveri S.V., Vassilev T.L. 2010. Exposure of $\mathrm{IgG}$ to an acidic environment results in molecular modifications and in enhanced protective activity in sepsis. FEBS J. 277, 3039-3050.

17. Djoumerska I., Tchorbanov A., Pashov A., Vassilev T. 2005. The autoreactivity of therapeutic intravenous immunoglobulin (IVIG) preparations depends on the fractionation methods used. Scand. J. Immunol. 61, 357-363.

18. McIntyre J.A., Faulk W.P. 2009. Redox-reactive autoantibodies: Biochemistry, characterization, and specificities. Clin. Rev. Allergy Immunol. 37, 49-54.

19. Dimitrov J.D., Roumenina L.T., Doltchinkova V.R., Mihaylova N.M., Lacroix-Desmazes S., Kaveri S.V., Vassilev T.L. 2007. Antibodies use heme as a cofactor to extend their pathogen elimination activity and to acquire new effector functions. J. Biol. Chem. 282, 26696-26706.

20. Iwashyna T.J., Ely E.W., Smith D.M., Langa K.M. 2010. Long-term cognitive impairment and functional disability among survivors of severe sepsis. J. Am. Med. Assoc. 304, 1787-1794.

21. Perl T.M., Dvorak L., Hwang T., Wenzel R.P. 1995. Long-term survival and function after suspected Gramnegative sepsis. J. Am. Med. Assoc. 274, 338-345.

22. Quartin A.A., Schein R.M., Kett D.H., Peduzzi P.N. 1997. Magnitude and duration of the effect of sepsis on survival. Department of veterans affairs systemic sepsis cooperative studies group. J. Am. Med. Assoc. 277, 1058-1063.

23. Zhang Q., Raoof M., Chen Y., Sumi Y., Sursal T., Junger W., Brohi K., Itagaki K., Hauser C.J. 2010. Circulating mitochondrial DAMPs cause inflammatory responses to injury. Nature. 464, 104-107.

24. Figueiredo R.T., Fernandez P.L., Mourao-Sa D.S., Porto B.N., Dutra F.F., Alves L.S., Oliveira M.F., Oliveira P.L., Graça-Souza A.V., Bozza M.T. 2007. Characterization of heme as activator of Toll-like receptor 4. J. Biol. Chem. 282, 20221-20229.

25. Xiao W., Mindrinos M.N., Seok J., et al. 2011. A genomic storm in critically injured humans. J. Exp. Med. 208, 2581-2590.

26. Soares M.O., Welton N.J., Harrison D.A., Peura P., Shankar-Hari M., Harvey S.E., Madan J.J., Ades A.E., Palmer S.J., Rowan K.M. 2012. An evaluation of the feasibility, cost and value of information of a multicentre randomised controlled trial of intravenous immunoglobulin for sepsis (severe sepsis and septic shock): Incorporating a systematic review, meta-analysis and value of information analysis. Health Technol. Assess. 16, 1-186.

27. Rimmelzwaan G.F., van Riel D., Baars M., Bestebroer T.M., van Amerongen G., Fouchier R.A., Osterhaus A.D., Kuiken T. 2006. Influenza A virus (H5N1) infection in cats causes systemic disease with potential novel routes of virus spread within and between hosts. Am. J. Pathol. 168, 176-183; quiz 364.

28. Ignatyev G., Steinkasserer A., Streltsova M., Atrasheuskaya A., Agafonov A., Lubitz W. 2000. Experi- 
mental study on the possibility of treatment of some hemorrhagic fevers. J. Biotechnol. 83, 67-76.

29. Atrasheuskaya A., Petzelbauer P., Fredeking T.M., Ignatyev G. 2003. Anti-TNF antibody treatment reduces mortality in experimental dengue virus infection. FEMS Immunol. Med. Microbiol. 35, 33-42.

30. Cheung C.Y., Poon L.L., Lau A.S., Luk W., Lau Y.L., Shortridge K.F., Gordon S., Guan Y., Peiris J.S. 2002. Induction of proinflammatory cytokines in human macrophages by influenza A (H5N1) viruses: A mechanism for the unusual severity of human disease? Lancet. 360, 1831-1837.

31. Dimitrov J.D., Ivanovska N.D., Lacroix-Desmazes S., Doltchinkova V.R., Kaveri S.V., Vassilev T.L. 2006. Ferrous ions and reactive oxygen species increase antigen-binding and anti-inflammatory activities of immunoglobulin G. J. Biol. Chem. 281, 439-446.

32. Mihaylova N.M., Dimitrov J.D., Djoumerska-Alexieva I.K., Vassilev T.L. 2008. Inflammation-induced enhancement of IgG immunoreactivity. Inflamm. Res. 57, $1-3$.

33. Djoumerska-Alexieva I., Roumenina L., Pashov A., Dimitrov J., Hadzhieva M., Lindig S., Voynova E., Dimitrova P., Ivanovska N., Bockmeyer C., Stefanova Z., Fitting C., Bläss M., Claus R., von Gunten S., et al. 2015. Intravenous immunoglobulin with enhanced polyspecificity improves survival in experimental sepsis and aseptic systemic inflammatory response syndromes. Mol. Med. 21 (1), 1002-1010.

34. Rauch P.J., Chudnovskiy A., Robbins C.S., Weber G.F., Etzrodt M., Hilgendorf I., Tiglao E., Figueiredo J.L., Iwamoto Y., Theurl I., Gorbatov R., Waring M.T., Chicoine A.T., Mouded M., Pittet M.J., et al. 2012. Innate response activator $\mathrm{B}$ cells protect against microbial sepsis. Science. 335, 597-601.

35. Coutinho A., Avrameas S. 1992. Speculations on immunosomatics: Potential diagnostic and therapeutic value of immune homeostasis concepts. Scand. J. Immunol. 36, 527-532.

36. Ochsenbein A.F., Fehr T., Lutz C., Suter M., Brombacher F., Hengartner H., Zinkernagel R.M. 1999. Control of early viral and bacterial distribution and disease by natural antibodies. Science. 286, 2156-2159.

37. Gunti S., Notkins A.L. 2015. Polyreactive antibodies: Function and quantification. J. Infect. Dis. 212 (Suppl. 1), S42-S46.

38. Sfriso P., Ghirardello A., Botsios C., Tonon M., Zen M., Bassi N., Bassetto F., Doria A. 2010. Infections and autoimmunity: the multifaceted relationship. J. Leukoc. Biol. 87, 385-395.

Translated by D. Timchenko 